Piotr KacPrzyK (Warszawa)

\title{
GLOBAL EXISTENCE FOR THE INFLOW-OUTFLOW PROBLEM FOR THE NAVIER-STOKES EQUATIONS IN A CYLINDER
}

Abstract. Global existence of regular solutions to the Navier-Stokes equations describing the motion of an incompressible viscous fluid in a cylindrical pipe with large inflow and outflow is shown. To prove the long time existence we need smallness of derivatives, with respect to the variable along the axis of the cylinder, of the external force and of the initial velocity in $L_{2}$-norms. Moreover, we need smallness of derivatives of inflow and outflow with respect to tangent directions to the boundary and with respect to time in some norms. The global existence is proved step by step using the existence on the time interval $[0, T]$, with $T$ sufficiently large.

1. Introduction. We consider viscous incompressible fluid motions in a finite cylinder with large inflow and outflow and under boundary slip conditions. The following initial-boundary value problem is examined:

$$
\begin{array}{ll}
v_{, t}+v \cdot \nabla v-\operatorname{div} \mathbb{T}(v, p)=f & \text { in } \Omega^{T}=\Omega \times(0, T), \\
\operatorname{div} v=0 & \text { in } \Omega^{T}, \\
v \cdot \bar{n}=0 & \text { on } S_{1}^{T}=S_{1} \times(0, T), \\
\nu \bar{n} \cdot \mathbb{D}(v) \cdot \bar{\tau}_{\alpha}+\gamma v \cdot \bar{\tau}_{\alpha}=0, \quad \alpha=1,2, & \text { on } S_{1}^{T}, \\
v \cdot \bar{n}=d & \text { on } S_{2}^{T}=S_{2} \times(0, T), \\
\bar{n} \cdot \mathbb{D}(v) \cdot \bar{\tau}_{\alpha}=0, \quad \alpha=1,2, & \text { on } S_{2}^{T}, \\
\left.v\right|_{t=0}=v(0) & \text { in } \Omega,
\end{array}
$$

where $\Omega \subset \mathbb{R}^{3}, S=S_{1} \cup S_{2}=\partial \Omega, v=v(x, t)=\left(v_{1}(x, t), v_{2}(x, t), v_{3}(x, t)\right)$ $\in \mathbb{R}^{3}$ is the velocity vector of the fluid motion, $p=p(x, t) \in \mathbb{R}^{1}$ the pressure,

2000 Mathematics Subject Classification: 35Q35, 76D03, 76D05.

Key words and phrases: Navier-Stokes equations, inflow-outflow problem, slip boundary conditions, cylindrical domains, global existence of regular solutions. 
$f=f(x, t)=\left(f_{1}(x, t), f_{2}(x, t), f_{3}(x, t)\right) \in \mathbb{R}^{3}$ the external force field, $\bar{n}$ the unit outward vector normal to the boundary $S$, and $\bar{\tau}_{\alpha}, \alpha=1,2$, are tangent vectors to $S$. Moreover, $\mathbb{T}(v, p)$ is the stress tensor of the form

$$
\mathbb{T}(v, p)=\nu \mathbb{D}(v)-p I,
$$

where $\nu$ is the constant viscosity coefficient, $I$ the unit matrix and $\mathbb{D}(v)$ the dilatation tensor

$$
\mathbb{D}(v)=\left\{v_{i, x_{j}}+v_{j, x_{i}}\right\}_{i, j=1,2,3} .
$$

Finally, $\gamma>0$ is the slip coefficient.

Here $\Omega \subset \mathbb{R}^{3}$ is a cylindrical type domain parallel to the $x_{3}$ axis with arbitrary cross section. We assume that $S_{1}$ is the part of the boundary which is parallel to the $x_{3}$ axis and $S_{2}$ is perpendicular to $x_{3}$. Hence

$$
\begin{aligned}
S_{1} & =\left\{x \in \mathbb{R}^{3}: \varphi\left(x_{1}, x_{2}\right)=c_{0},-a<x_{3}<a\right\}, \\
S_{2}(-a) & =\left\{x \in \mathbb{R}^{3}: \varphi\left(x_{1}, x_{2}\right)<c_{0}, x_{3}=-a\right\}, \\
S_{2}(a) & =\left\{x \in \mathbb{R}^{3}: \varphi\left(x_{1}, x_{2}\right)<c_{0}, x_{3}=a\right\},
\end{aligned}
$$

where $a, c_{0}$ are given positive numbers and $\varphi\left(x_{1}, x_{2}\right)=c_{0}$ describes a sufficiently smooth closed curve in the plane $x_{3}=$ const.

To describe inflow and outflow we define

$$
d_{1}=-\left.v \cdot \bar{n}\right|_{S_{2}(-a)}, \quad d_{2}=\left.v \cdot \bar{n}\right|_{S_{2}(a)},
$$

so $d_{i} \geq 0, i=1,2$, and by $(1.1)_{2,3}$ and (1.2) we have the compatibility condition

$$
\Phi \equiv \int_{S_{2}(-a)} d_{1} d S_{2}=\int_{S_{2}(a)} d_{2} d S_{2}
$$

where $\Phi$ is the flux.

Let us introduce an extension $\alpha=\alpha(x, t) \in \mathbb{R}$ such that

$$
\left.\alpha\right|_{S_{2}(-a)}=d_{1},\left.\quad \alpha\right|_{S_{2}(a)}=d_{2} .
$$

Then equations (1.1) $2,3,6$ and (1.3) imply the compatibility condition

$$
\int_{\Omega} \alpha_{, x_{3}} d x=-\left.\int_{S_{2}(-a)} \alpha\right|_{x_{3}=-a} d S_{2}+\left.\int_{S_{2}(a)} \alpha\right|_{x_{3}=a} d S_{2}=0 .
$$

The aim of this paper is to show the global in time existence of regular solutions to (1.1). We base on [1], where the existence of a regular solution for large time has been proved by the Leray-Schauder fixed point theorem.

The proof of global existence in [1] is not explicit. It depends on showing that for a long time solution the norms $\|v(t)\|_{L_{2}(\Omega)},\|h(t)\|_{L_{2}(\Omega)}$ decrease with time. Therefore for $T$ sufficiently large we have

$$
\|v(T)\|_{L_{2}(\Omega)} \leq\|v(0)\|_{L_{2}(\Omega)}, \quad\|h(T)\|_{L_{2}(\Omega)} \leq\|h(0)\|_{L_{2}(\Omega)} .
$$


Hence, (1.5) and appropriate estimates of data functions $(f, g, d)$ imply prolongation of a local solution from the interval $[0, T]$.

Hence, global existence follows.

In this paper the proof of global existence is explicit because we control all estimates step by step. Moreover, we show that

$$
\|v(k T)\|_{L_{2}(\Omega)} \leq\|v(0)\|_{L_{2}(\Omega)}, \quad \forall k \in \mathbb{N} .
$$

Using the existence of solution on $[0, T]$ we inductively prolong it on $\mathbb{R}_{+}$ by employing some cut-off functions. A similar technique has been used in $[5,6]$.

To formulate the main result we need the notation (see Section 2 for definitions of norms)

$$
\begin{aligned}
\Gamma^{2}(k T, t)= & |\alpha|_{2, S_{1}}^{2}+\left|\alpha_{, t}\right|_{6 / 5, \Omega}^{2}+\left|\alpha_{, x_{3}}\right|_{6 / 5, \Omega}^{2}+\left(1+\|\alpha\|_{1,3, \Omega}^{2}\right)|\nabla \alpha|_{2, \Omega}^{2} \\
& +|f|_{6 / 5, \Omega}^{2}, \\
\Gamma_{1}^{2}(k T, t)= & \|\alpha\|_{L_{\infty}\left(k T, t L_{2}(\Omega)\right)}^{2}+\left\|\alpha_{, x_{3}}\right\|_{L_{\infty}\left(k T, t ; L_{2}(\Omega)\right)}^{2}+\int_{k T}^{t}\left\|\alpha\left(t^{\prime}\right)\right\|_{1,2, \Omega}^{2} d t^{\prime}, \\
l_{1}^{2}(k T, t)= & c \exp c\left(\left|d_{1}\right|_{3,6, S_{2} \times(k T, t)}^{6}+|\nabla \alpha|_{3,2, \Omega \times(k T, t)}^{2}\right) \\
& \cdot\left(\int_{k T}^{t} \Gamma^{2}\left(t^{\prime}\right) d t^{\prime}+\Gamma_{1}^{2}(k T, t)+|v(k T)|_{2, \Omega}^{2}\right), \\
G(k T, t)= & l_{1}(k T, t)+\left\|d_{1}\right\|_{3 / 2,2, S_{2} \times(k T, t)}+|f|_{2, \Omega \times(k T, t)} \\
& +\left|F_{3}\right|_{10 / 7, \Omega \times(k T, t)}+\left|d_{1}\right|_{\infty, \Omega \times(k T, t)}, \quad \text { where } \quad F_{3}=(\operatorname{rot} v)_{3}, \\
G^{\prime}(k T, t)= & |g|_{2, \Omega \times(k T, t)}+l_{1}(k T, t)+\left\|d_{1, x^{\prime}}\right\|_{3 / 2,2, S_{2} \times(k T, t),},
\end{aligned}
$$

where $g=f_{, x_{3}}$, and

$$
\begin{aligned}
\eta(k T, t)= & \left\|d_{1, x^{\prime}}\right\|_{L_{\infty}\left(k T, t ; H^{1}\left(S_{2}\right)\right)}+\left\|d_{1, t}\right\|_{L_{2}\left(k T, t ; H^{1}\left(S_{2}\right)\right)} \\
& +\left\|f_{3}\right\|_{L_{2}\left(k T, t ; L_{4 / 3}(\Omega)\right)}+\|g\|_{L_{2}\left(k T, t ; L_{6 / 5}(\Omega)\right)}+\frac{1}{T} l_{1}(k T, t),
\end{aligned}
$$

where $t \in(k T,(k+1) T)$.

Theorem 1.1 (global existence). Assume that $t \in(k T,(k+1) T), k \in$ $\mathbb{N} \cup\{0\} \equiv \mathbb{N}_{0}$,

$$
\begin{aligned}
& \int_{k T}^{t} \Gamma^{2}\left(k T, t^{\prime}\right) d t^{\prime} \leq \int_{0}^{T} \Gamma^{2}\left(0, t^{\prime}\right) d t^{\prime}, \\
& \Gamma_{1}(k T, t) \leq \Gamma_{1}(0, T), \quad G(k T, t) \leq G(0, T), \quad G^{\prime}(k T, t) \leq G^{\prime}(0, T), \\
& l_{1}(k T, t) \leq l_{1}(0, T), \quad \eta(k T, t) \leq \eta(0, T) .
\end{aligned}
$$


Assume that $\eta(k T,(k+1) T)$ is so small that there exists a positive constant A such that

$$
\varphi(A, G(k T, t)) \eta(k T, t)+G^{\prime}(k T, t)<A,
$$

where $\varphi$ is some positive increasing function. Then there exists a solution to problem (1.1) such that

$$
\begin{aligned}
& \left\|v_{, x_{3}}\right\|_{W_{2}^{2,1}((k T,(k+1) T) \times \Omega)} \leq A, \\
& \|v\|_{W_{2}^{2,1}((k T,(k+1) T) \times \Omega)} \leq c\left(A^{2}+1\right),
\end{aligned}
$$

where $A>0$ is a constant chosen for a given $T$ and independent of $k \in \mathbb{N}$.

Remark. A global solution to problem (1.1) exists if $d_{1} \rightarrow 0$ as $t \rightarrow \infty$.

In $[9,11,12]$ the existence of global regular solutions which are close to axially symmetric solutions is proved in axially symmetric domains by the method of successive approximations. The main step in the proofs of $[11,12]$ is the proof of an estimate and the existence of solutions to a problem for $\chi=(\operatorname{rot} v)_{\varphi}$ with appropriate boundary conditions such that $\left.\chi\right|_{S}$ depends on $\left.v\right|_{S}$ only (and not on $\left.v_{, x}\right|_{S}$ ).

In $[5,6,9,10]$ the long time existence of solutions which are close to $2 \mathrm{~d}$ solutions is proved in non-axially cylindrical domains. In $[7,10]$ the existence is proved in Besov spaces, and in [6] the proof is simplified, so it is done in Sobolev spaces only. In [5] global existence by prolongation of long time solution from [6] is proved. In [10] the inflow-outflow problem is considered by using Besov spaces.

In this paper the proof of global existence is simplified because the Besov spaces are replaced by Sobolev spaces. This makes the proof totally different and much clearer.

As we examine the inflow-outflow problem, all proofs in this paper are different from the corresponding proofs in [12] because the expressions involving the inflow-outflow flux must be additionally estimated. Moreover, in this case we consider problems with nonhomogeneous boundary conditions, so our approach must be different.

In $[6,7,9,10]$ the Leray-Schauder fixed point theorem was applied. We mention that the present paper generalizes the result from [5] to the inflow-outflow case and simplifies the proof from [10].

We underline that the main step in the proofs of long time existence in $[6,7,9,10]$ is an estimate for the component $\chi=(\operatorname{rot} v)_{3}$ of the vorticity, which is possible because the slip boundary conditions are assumed. 
2. Notation and auxiliary results. To simplify the presentation we introduce the following notation:

$$
\begin{array}{ll}
|u|_{p, Q}=\|u\|_{L_{p}(Q)}, & Q \in\left\{\Omega^{T}, S^{T}, \Omega, S\right\}, p \in[1, \infty], \\
\|u\|_{s, Q}=\|u\|_{H^{s}(Q)}, & Q \in\{\Omega, S\}, s \in \mathbb{R}_{+} \cup\{0\}, \\
\|u\|_{s, Q^{T}}=\|u\|_{W_{2}^{s, s / 2}\left(Q^{T}\right)}, & Q \in\{\Omega, S\}, s \in \mathbb{R}_{+} \cup\{0\}, \\
|u|_{p, q, Q^{T}}=\|u\|_{L_{q}\left(0, T ; L_{p}(Q)\right)}, & Q \in\{\Omega, S\}, p, q \in[1, \infty], \\
\|u\|_{s, q, Q^{T}}=\|u\|_{W_{q}^{s, s / 2}\left(Q^{T}\right)}, & Q \in\{\Omega, S\}, s \in \mathbb{R}_{+} \cup\{0\}, q \in[1, \infty], \\
\|u\|_{s, q, Q}=\|u\|_{W_{q}^{s}(Q)}, & Q \in\{\Omega, S\}, s \in \mathbb{R}_{+} \cup\{0\}, q \in[1, \infty] .
\end{array}
$$

By $c$ we denote a generic constant which changes its magnitude from formula to formula. By $\bar{c}(\sigma)$ and $\varphi(\sigma)$ we understand generic functions which are always positive and increasing. Finally, we do not distinguish scalar and vector-valued functions in notation.

We introduce the space

$$
\begin{aligned}
V_{2}^{k}\left(\Omega^{T}\right)=\left\{u:\|u\|_{V_{2}^{k}\left(\Omega^{T}\right)}=\right. & \operatorname{esssup}_{t \in(0, T)}\|u\|_{H^{k}(\Omega)} \\
& \left.+\left(\int_{0}^{T}\|\nabla u(t)\|_{H^{k}(\Omega)}^{2} d t\right)^{1 / 2}<\infty\right\}, \quad k \in \mathbb{N} .
\end{aligned}
$$

From [10] we get the following result for weak solutions to problem (1.1):

Lemma 2.1. Assume that $d_{1} \in L_{6}\left(0, T ; L_{3}\left(S_{2}\right)\right), \nabla \alpha \in L_{2}\left(0, T ; L_{3}(\Omega)\right)$, $\int_{0}^{T} \Gamma^{2}(t) d t<\infty$, where

$$
\begin{aligned}
\Gamma^{2}(t)= & |\alpha|_{2, S_{1}}^{2}+\left|\alpha_{, t}\right|_{6 / 5, \Omega}^{2}+\left|\alpha_{, x_{3} t}\right|_{6 / 5, \Omega}^{2}+\left(1+\|\alpha\|_{1,3, \Omega}^{2}\right)|\nabla \alpha|_{2, \Omega}^{2} \\
& +|f|_{6 / 5, \Omega}^{2},
\end{aligned}
$$

and

$$
\Gamma_{1}^{2}(T)=|\alpha|_{2, \infty, \Omega}^{2}+\left|\alpha_{, x_{3}}\right|_{2, \infty, \Omega^{T}}^{2}+\int_{0}^{T}\|\alpha(t)\|_{1,2}^{2} d t<\infty .
$$

Then

$$
\begin{aligned}
& \|v\|_{L_{\infty}\left(0, T ; L_{2}(\Omega)\right)}^{2}+\|v\|_{L_{2}\left(0, T ; H^{1}(\Omega)\right)}^{2} \\
& \leq c \exp \left(c\left(\left|d_{1}\right|_{3,6, S_{2}^{T}}^{6}+|\nabla \alpha|_{3,2, \Omega^{T}}^{2}\right)\right)\left(\int_{0}^{T} \Gamma^{2}(t) d t+\Gamma_{1}^{2}(T)+|v(0)|_{2, \Omega}^{2}\right) \\
& \equiv l_{1}^{2}(0, T) .
\end{aligned}
$$

From [10, (2.13)] we get

$$
\frac{d}{d t}|w|_{2, \Omega}^{2} \leq c\left(\left|d_{1}\right|_{3, S_{2}}^{6}+|\nabla \alpha|_{3, \Omega}^{2}-\nu\right)|w|_{2, \Omega}^{2}+c \Gamma^{2}(t) .
$$


From (2.3) we obtain

$$
\begin{aligned}
|w(t)|_{2, \Omega}^{2} \leq & \exp \left(c\left(\left|d_{1}\right|_{3,6, S_{2}^{t}}^{6}+|\nabla \alpha|_{3,2, \Omega^{t}}^{2}-\nu t\right)\right) \\
& \cdot\left(c \int_{0}^{t} \Gamma^{2}\left(t^{\prime}\right) d t^{\prime}+|w(0)|_{2, \Omega}^{2}\right) .
\end{aligned}
$$

If $T$ is sufficiently large, then $|w(T)|_{2, \Omega} \leq|w(0)|_{2, \Omega}$ and from [10, (1.7)] we obtain $|v(T)|_{2, \Omega} \leq|v(0)|_{2, \Omega}$. For $t \in(k T,(k+1) T)$ assume that

$$
\left|d_{1}\right|_{3,6, S_{2}^{T}}^{6}+|\nabla \alpha|_{3,2, \Omega^{T}}^{2} \geq\left\|d_{1}\right\|_{L_{6}\left(k T, t ; L_{3}\left(S_{2}\right)\right)}^{6}+\|\nabla \alpha\|_{L_{2}\left(k T, t ; L_{3}(\Omega)\right)}^{2},
$$

$$
\int_{0}^{T} \Gamma^{2}(t) d t \geq \int_{k T}^{t} \Gamma^{2}\left(t^{\prime}\right) d t^{\prime}
$$

Repeating the above considerations we obtain

$$
\mid v(k+1) T)\left.\right|_{2, \Omega} \leq|v(k T)|_{2, \Omega}, \quad k \in \mathbb{N}
$$

From (2.5) we have, for $t \in(k T,(k+1) T)$,

$$
\begin{aligned}
& |\alpha|_{2, \infty, \Omega^{T}}^{2}+\left|\alpha_{, x_{3}}\right|_{2, \infty, \Omega^{T}}^{2}+\int_{0}^{T}\|\alpha(t)\|_{1, \Omega}^{2} d t \\
& \geq\|\alpha\|_{L_{\infty}\left(k T, t ; L_{2}(\Omega)\right)}^{2}+\left\|\alpha_{, x_{3}}\right\|_{L_{\infty}\left(k T, t ; L_{2}(\Omega)\right)}^{2}+\int_{k T}^{t}\left\|\alpha\left(t^{\prime}\right)\right\|_{1, \Omega}^{2} d t^{\prime} .
\end{aligned}
$$

Then repeating the considerations from [10, Lemma 2.3] in the interval $(k T,(k+1) T)$ and using $(2.6)$ we obtain, for $t \in(k T,(k+1) T)$,

$$
\|v(t)\|_{L_{2}(\Omega)}^{2}+\int_{k T}^{t}\left\|v\left(t^{\prime}\right)\right\|_{H^{1}(\Omega)}^{2} d t^{\prime} \leq l_{1}^{2}(0, T) .
$$

This concludes the proof.

Finally, we introduce the quantities

$$
h=v_{, x_{3}}, \quad q=p_{, x_{3}}, \quad g=f_{, x_{3}}, \quad w=v_{3}, \quad \chi=v_{2, x_{1}}-v_{1, x_{2}} .
$$

3. Basic formulations. To prove the existence of global solutions to problem (1.1) we follow [10]. Therefore we need problems for the quantities (2.9). First, from [10] we have 
Lemma 3.1. The quantities $h, q$ are solutions to the problem

$$
\begin{array}{ll}
h_{, t}-\operatorname{div} \mathbb{T}(h, q)=-v \cdot \nabla h-h \cdot \nabla v+g & \text { in } \Omega^{T}, \\
\operatorname{div} h=0 & \text { in } \Omega^{T}, \\
\bar{n} \cdot h=0 & \text { on } S_{1}^{T}, \\
\nu \bar{n} \cdot \mathbb{D}(h) \cdot \bar{\tau}_{\alpha}+\gamma h \cdot \bar{\tau}_{\alpha}=0, \quad \alpha=1,2, & \text { on } S_{1}^{T}, \\
h_{i}=-d_{, x_{i}}, \quad i=1,2, & \text { on } S_{2}^{T}, \\
h_{3, x_{3}}=\Delta^{\prime} d & \text { on } S_{2}^{T}, \\
h_{t=0}=h(0) & \text { in } \Omega,
\end{array}
$$

where $\Delta^{\prime}=\partial_{x_{1}}^{2}+\partial_{x_{2}}^{2}$, $d$ replaces $d_{1}$ and $d_{2}$, because $\left.d\right|_{S_{2}(-a)}=d_{1},\left.d\right|_{S_{2}(a)}=d_{2}$.

Proof. Equations (3.1) 1,2,3,4,7 follow directly from the corresponding equations in (1.1) by differentiation with respect to $x_{3}$, because $S_{1}$ is parallel to the $x_{3}$ axis.

To show $(3.1)_{5,6}$ we recall that

$$
\left.v_{3}\right|_{S_{2}}=d,\left.\quad\left(v_{i, x_{3}}+v_{3, x_{i}}\right)\right|_{S_{2}}=0, \quad i=1,2 .
$$

Hence $\left.v_{i, x_{3}}\right|_{S_{2}}=-d_{, x_{i}}, i=1,2$, and $(3.1)_{5}$ holds.

From $(1.1)_{2}$ we have $\left.v_{3, x_{3} x_{3}}\right|_{S_{2}}=-\left.\left(v_{1, x_{3} x_{1}}+v_{2, x_{3} x_{2}}\right)\right|_{S_{2}}=d_{, x_{1} x_{1}}+d_{, x_{2} x_{2}}=$ $\Delta^{\prime} d$. Hence $(3.1)_{6}$ follows. This ends the proof.

Lemma 3.2. The function $\chi=v_{2, x_{1}}-v_{1, x_{2}}$ is a solution to the problem

$$
\begin{aligned}
& \chi_{, t}+v \cdot \nabla \chi-h_{3} \chi+h_{2} w_{, x_{1}}-h_{1} w_{x_{2}}-\nu \Delta \chi=F_{3} \quad \text { in } \Omega^{T}, \\
& \left.\chi\right|_{S_{1}}=-v_{i}\left(n_{i, x_{j}} \tau_{1 j}+\tau_{1 i, x_{j}} n_{j}\right)+\frac{\gamma}{\nu} v_{j} \tau_{1 j} \\
& +v \cdot \bar{\tau}_{1}\left(\tau_{12, x_{1}}-\tau_{11, x_{2}}\right) \equiv \chi_{*} \quad \text { on } S_{1}^{T}, \\
& \chi_{, x_{3}}=0 \\
& \text { on } S_{2}^{T} \text {, } \\
& \left.\chi\right|_{t=0}=\chi(0) \\
& \text { in } \Omega \text {, }
\end{aligned}
$$

where

$$
\begin{gathered}
\left.\bar{n}\right|_{S_{1}}=\frac{\left(\varphi, x_{1}, \varphi, x_{2}, 0\right)}{\sqrt{\varphi_{, x_{1}}^{2}+\varphi_{, x_{2}}^{2}}},\left.\quad \bar{\tau}_{1}\right|_{S_{1}}=\frac{\left(-\varphi_{, x_{2}}, \varphi_{, x_{1}}, 0\right)}{\sqrt{\varphi_{, x_{1}}^{2}+\varphi_{, x_{2}}^{2}}},\left.\quad \bar{\tau}_{2}\right|_{S_{1}}=(0,0,1) \equiv \bar{e}_{3}, \\
\left.\bar{n}\right|_{S_{2}}=\bar{e}_{3},\left.\quad \bar{\tau}_{1}\right|_{S_{2}}=\bar{e}_{1},\left.\quad \bar{\tau}_{2}\right|_{S_{2}}=\bar{e}_{2},
\end{gathered}
$$

where $\bar{e}_{1}=(1,0,0), \bar{e}_{2}=(0,1,0)$ and $F_{3}=f_{2, x_{1}}-f_{1, x_{2}}$.

Proof. Differentiating the first equation of $(1.1)_{1}$ with respect to $x_{2}$, the second equation of $(1.1)_{1}$ with respect to $x_{1}$, and subtracting the results yields $(3.3)_{1}$. 
To show $(3.3)_{2}$ we extend the vectors $\bar{\tau}_{1}, \bar{n}$ to a neighbourhood of $S_{1}$. In this neighbourhood $v^{\prime}=\left(v_{1}, v_{2}\right)$ can be expressed in the form

$$
v^{\prime}=v \cdot \bar{\tau}_{1} \bar{\tau}_{1}+v \cdot \bar{n} \bar{n}
$$

Then

$$
\begin{aligned}
\left.\chi\right|_{S_{1}} & =\left.\left[\left(v \cdot \bar{\tau}_{1} \tau_{12}+v \cdot \bar{n} n_{2}\right)_{, x_{1}}-\left(v \cdot \bar{\tau}_{1} \tau_{11}+v \cdot \bar{n} n_{1}\right)_{, x_{2}}\right]\right|_{S_{1}} \\
& =\left.\left[-\bar{n} \cdot \nabla\left(v \cdot \bar{\tau}_{1}\right)+v \cdot \bar{\tau}_{1}\left(\tau_{12, x_{1}}-\tau_{11, x_{2}}\right)\right]\right|_{S_{1}},
\end{aligned}
$$

where $(1.1)_{3}$ was employed and $\tau_{1 i}, n_{i}$ are the $i$ th Cartesian coordinates.

Utilizing (1.1) ${ }_{3}$ in $(1.1)_{4}$ for $\alpha=1$ yields

$$
\nu \bar{n} \cdot \nabla\left(v \cdot \bar{\tau}_{1}\right)-\nu v_{i}\left(n_{i, x_{j}} \tau_{1 j}+\tau_{1 i, x_{j}} n_{j}\right)+\gamma v \cdot \bar{\tau}_{1}=0 .
$$

Employing (3.5) in (3.4) yields $(3.3)_{2}$. By the definition of $\chi$ and $(3.1)_{5}$ we have

$$
\left.\chi_{, x_{3}}\right|_{S_{2}}+\left.\left(v_{2, x_{1} x_{3}}-v_{1, x_{2} x_{3}}\right)\right|_{S_{2}}=-\left.\left(d_{, x_{1} x_{2}}-d_{, x_{2} x_{1}}\right)\right|_{S_{2}}=0 .
$$

This ends the proof.

For solutions to problem (3.1) we get (see [10])

Lemma 3.3. Assume that $v$ is a weak solution to problem (1.1). Then

$$
\begin{aligned}
& \sup _{t<T}|h(t)|_{2, \Omega}^{2}+\nu \int_{0}^{T}\|h(t)\|_{1, \Omega}^{2} d t+\gamma\left|h \cdot \bar{\tau}_{\alpha}\right|_{2, S_{1}^{T}}^{2} \\
& \leq \varphi\left(\left|d_{1}\right|_{3,6, S_{2}^{T}},|\nabla v|_{3,2, \Omega^{T}}, l_{1}(0, T),\left|d_{1}\right|_{3, \infty, S_{2}^{T}}\right) \eta_{1}^{2}(T),
\end{aligned}
$$

where $\varphi$ is an increasing positive function, $l_{1}$ is defined by (2.7) and

$$
\begin{aligned}
\eta_{1}(t)= & \sup _{t^{\prime} \leq t}\left\|d_{1, x^{\prime}}\left(t^{\prime}\right)\right\|_{1, S_{2}}+\left\|d_{1, x^{\prime}}\right\|_{L_{2}\left(0, t ; H^{1}\left(S_{2}\right)\right)} \\
& +\left\|d_{1, t}\right\|_{L_{2}\left(0, t ; W_{6 / 5}^{1}\left(S_{2}\right)\right)}+\left|f_{3}\right|_{4 / 3,2, S_{2}^{t}}+|g|_{6 / 5,2, \Omega^{t}}+|h(0)|_{2, \Omega} .
\end{aligned}
$$

4. Estimates. In our estimates we will use cut-off functions $\xi^{\left(k_{n}\right)} \in$ $C_{0}^{\infty}(0, \infty)$ with

$$
\xi^{\left(k_{n}\right)}(t)= \begin{cases}1 & \text { for } t \in((k-n) T,(k+1) T), \\ 0 & \text { for } t \leq(k-n-1) T\end{cases}
$$

and $\xi_{, t}^{\left(k_{n}\right)} \leq 1 / T$. Moreover, from now on let $u^{\left(k_{n}\right)}=\xi^{\left(k_{n}\right)} u$. 
Using the notation we obtain from (3.1) the problem

$$
\begin{array}{ll}
h_{, t}^{\left(k_{n}\right)}-\operatorname{div} \mathbb{T}\left(h^{\left(k_{n}\right)}, q^{\left(k_{n}\right)}\right)=-v \cdot \nabla h^{\left(k_{n}\right)}-h^{\left(k_{n}\right)} \cdot \nabla v & \\
\quad+g^{\left(k_{n}\right)}+\xi_{, t}^{\left(k_{n}\right)} h & \text { in } \Omega^{t}, \\
\operatorname{div} h^{\left(k_{n}\right)}=0 & \text { in } \Omega^{t}, \\
h^{\left(k_{n}\right)} \cdot n=d^{\left(k_{n}\right)} & \text { on } S_{1}^{t}, \\
n \cdot \mathbb{T}\left(h^{\left(k_{n}\right)}, q^{\left(k_{n}\right)}\right) \cdot \tau_{\alpha}=0, \quad \alpha=1,2, & \text { on } S_{1}^{t}, \\
h_{i}^{\left(k_{n}\right)}=0, \quad i=1,2, \quad h_{3, x_{3}}^{\left(k_{n}\right)}=0 & \text { on } S_{2}^{t}, \\
\left.h^{\left(k_{n}\right)}\right|_{t=(k-n-1) T}=0 & \text { in } \Omega,
\end{array}
$$

in the interval $((k-n-1) T,(k+1) T)$. In view of the energy type inequality [10, Lemma 3.7] we obtain, for $t \in((k-n-1) T,(k+1) T)$,

$$
\begin{gathered}
\sup _{t}\left|h^{\left(k_{n}\right)}(t)\right|_{2, \Omega}^{2}+\nu \int_{(k-n-1) T}^{t}\left\|h^{\left(k_{n}\right)}\left(t^{\prime}\right)\right\|_{1, \Omega}^{2} d t^{\prime}+\gamma\left|h^{\left(k_{n}\right)} \cdot \bar{\tau}_{\alpha}\right|_{2, S_{1}^{t}}^{2} \\
\leq \varphi\left(\left|d_{1}^{\left(k_{n}\right)}\right|_{3,6, S_{2}^{t}},\left|\nabla v^{\left(k_{n+1}\right)}\right|_{3,2, \Omega^{t}}, l_{1}(t),\left|d_{1}^{\left(k_{n}\right)}\right|_{3, \infty, S_{2}^{t}}\right) \\
\cdot \eta_{k_{n}}^{2}((k-n-1) T, t),
\end{gathered}
$$

where

$$
\begin{aligned}
\eta_{k_{n}}(( & -n-1) T, t)=\left\|d_{1, x^{\prime}}^{\left(k_{n}\right)}\right\|_{L_{\infty}\left((k-n-1) T, t ; H^{1}\left(S_{2}\right)\right)} \\
& +\left\|d_{1, t} \xi^{\left(k_{n}\right)}\right\|_{L_{2}\left((k-n-1) T, t, H^{1}\left(S_{2}\right)\right)}+\left\|d_{1, x^{\prime}}^{\left(k_{n}\right)}\right\|_{L_{2}\left((k-n-1) T, t ; H^{1}\left(S_{2}\right)\right)} \\
& +\left|f_{3}^{\left(k_{n}\right)}\right|_{4 / 3,2, \Omega^{t}}+\left|g^{\left(k_{n}\right)}\right|_{6 / 5,2, \Omega^{t}}+\frac{1}{T} l_{1}((k-n-1) T, t) .
\end{aligned}
$$

First we examine problem (3.3). Let $\tilde{\chi}$ be a solution of the problem

$$
\begin{array}{ll}
\tilde{\chi}_{, t}-\nu \Delta \tilde{\chi}=0 & \text { in } \Omega^{T}, \\
\tilde{\chi}=\chi_{*} & \text { on } S_{1}^{T}, \\
\tilde{\chi}_{, x_{3}}=0 & \text { on } S_{2}^{T}, \\
\left.\tilde{\chi}\right|_{t=0}=0 & \text { in } \Omega .
\end{array}
$$

Then $\chi^{\prime}=\chi-\tilde{\chi}$ satisfies

$$
\begin{array}{ll}
\chi_{, t}^{\prime}+v \cdot \nabla \chi^{\prime}-h_{3}\left(v_{2, x_{1}}-v_{1, x_{2}}\right)+h_{2} w_{, x_{1}}-h_{1} w_{, x_{2}} & \\
\quad-\nu \Delta \chi^{\prime}=F_{3}-v \cdot \nabla \tilde{\chi} & \text { in } \Omega^{T}, \\
\chi^{\prime}=0 & \text { on } S_{1}^{T}, \\
\chi_{, x_{3}}^{\prime}=0 & \text { on } S_{2}^{T}, \\
\left.\chi^{\prime}\right|_{t=0}=\chi(0) & \text { in } \Omega .
\end{array}
$$


LEMma 4.1. Assume that $h^{\left(k_{n}\right)} \in L_{5}(((k-n-1) T, t) \times \Omega), F_{3}^{\left(k_{n}\right)} \in$ $L_{10 / 7}(((k-n-1) T, t) \times \Omega), v^{\left(k_{n}\right)} \in W_{r}^{s, s / 2}(((k-n-1) T, t) \times \Omega)$ with $5 / r-3 / 2 \leq s$. Assume also that $v$ is a weak solution satisfying (2.8). Then

$$
\begin{aligned}
&\left|\chi^{\left(k_{n}\right)}(t)\right|_{2, \Omega}^{2}+\int_{(k-n-1) T}^{t}\left\|\chi^{\left(k_{n}\right)}\left(t^{\prime}\right)\right\|_{1, \Omega}^{2} d t^{\prime} \\
& \leq c\left(l_{1}^{2}((k-n-1) T, t)\left(\left|v^{\prime\left(k_{n}\right)}\right|_{3, \infty, S_{1}^{t}}^{2}+\left|h^{\left(k_{n}\right)}\right|_{5, \Omega^{t}}\right)\right. \\
&\left.+\left(1+\left\|d_{1}\right\|_{L_{\infty}\left(((k-n-1) T, t) \times S_{2}\right)}^{2}\right)\left\|v^{\prime\left(k_{n}\right)}\right\|_{s, r, \Omega^{t}}^{2}+\left|F_{3}^{\left(k_{n}\right)}\right|_{10 / 7, \Omega^{t}}^{2}\right),
\end{aligned}
$$

where $v^{\prime}=\left(v_{1}, v_{2}\right)$.

Proof. Multiplying $(4.4)$ by $\xi^{\left(k_{n}\right)}$, then by $\chi^{\prime\left(k_{n}\right)}$, and integrating the result over $\Omega$ we get

$$
\begin{aligned}
\frac{1}{2} \frac{d}{d t}\left|\chi^{\prime\left(k_{n}\right)}(t)\right|_{2, \Omega}^{2} & +\left|\nabla \chi^{\prime\left(k_{n}\right)}\right|_{2, \Omega}^{2}=\int_{\Omega}\left(v_{2, x_{1}}-v_{1, x_{2}}\right) h_{3}^{\left(k_{n}\right)} \chi^{\prime\left(k_{n}\right)} d x \\
& -\int_{\Omega}\left(h_{2}^{\left(k_{n}\right)} w_{, x_{1}}-h_{1}^{\left(k_{n}\right)} w_{, x_{2}}\right) \chi^{\prime\left(k_{n}\right)} d x+\int_{\Omega} v \cdot \nabla \tilde{\chi}^{\left(k_{n}\right)} \chi^{\prime\left(k_{n}\right)} d x \\
& +\int_{\Omega} F_{3}^{\left(k_{n}\right)} \chi^{\prime\left(k_{n}\right)} d x+\int_{\Omega} \xi_{, t}^{\left(k_{n}\right)} \chi^{\prime} \chi^{\prime\left(k_{n}\right)} d x .
\end{aligned}
$$

Utilizing the Poincaré inequality and integrating with respect to time for $t \in((k-n-1) T,(k+1) T)$ yields

$$
\begin{aligned}
\left|\chi^{\prime\left(k_{n}\right)}(t)\right|_{2, \Omega}^{2}+ & \int_{(k-n-1) T}^{t}\left\|\chi^{\prime\left(k_{n}\right)}\left(t^{\prime}\right)\right\|_{1, \Omega}^{2} d t^{\prime} \\
\leq & c\left(\int_{\Omega^{t}}\left|h_{3}^{\left(k_{n}\right)}\right|\left|\nabla v^{\prime}\right|\left|\chi^{\prime\left(k_{n}\right)}\right| d x d t\right. \\
& +\int_{\Omega^{t}}\left|h^{\prime\left(k_{n}\right)}\right|\left|\nabla^{\prime} w\right|\left|\chi^{\prime\left(k_{n}\right)}\right| d x d t \\
& +\left|\int_{(k-n-1) T} \int_{\Omega} v\left(t^{\prime}\right) \cdot \nabla \tilde{\chi}^{\left(k_{n}\right)}\left(t^{\prime}\right) \chi^{\prime\left(k_{n}\right)}\left(t^{\prime}\right) d x d t\right| \\
& \left.+\int_{\Omega^{t}}\left|F_{3}^{\left(k_{n}\right)}\right|\left|\chi^{\prime\left(k_{n}\right)}\right| d x d t+\frac{1}{T^{2}}\left\|\chi^{\prime}\right\|_{L_{2}(((k-n-1) T, t) \times \Omega)}\right) .
\end{aligned}
$$

We estimate the first term on the r.h.s. of (4.6) by

$$
\left|h_{3}^{\left(k_{n}\right)}\right|_{5, \Omega^{t}} \|\left.\nabla v^{\prime}\right|_{L_{2}(((k-n-1) T, t) \times \Omega)}\left|\chi^{\prime\left(k_{n}\right)}\right|_{10 / 3, \Omega^{t}}
$$


and the second by

$$
\left|h^{\prime\left(k_{n}\right)}\right|_{5, \Omega^{t}}\left\|\nabla^{\prime} w\right\|_{L_{2}(((k-n-1) T, t) \times \Omega)}\left|\chi^{\prime\left(k_{n}\right)}\right|_{10 / 3, \Omega^{t}},
$$

where $\nabla^{\prime}=\left(\partial_{x_{1}}, \partial_{x_{2}}\right)$.

The third term on the r.h.s. of (4.6) can be expressed in the form

$$
\begin{aligned}
& \mid \int_{(k-n-1) T} \int_{\Omega} v\left(t^{\prime}\right) \cdot \nabla \chi^{\prime\left(k_{n}\right)}\left(t^{\prime}\right) \tilde{\chi}^{\left(k_{n}\right)}\left(t^{\prime}\right) d x d t^{\prime} \\
& +\int_{(k-n-1) T}^{t} \int_{S_{2}} d\left(t^{\prime}\right) \chi^{\prime\left(k_{n}\right)}\left(t^{\prime}\right) \tilde{\chi}^{\left(k_{n}\right)}\left(t^{\prime}\right) d S_{2} d t^{\prime} \mid
\end{aligned}
$$

and estimated by

$$
\begin{aligned}
\varepsilon\left|\nabla \chi^{\prime\left(k_{n}\right)}\right|_{2, \Omega^{t}}^{2}+|\nabla v|_{L_{2}(((k-n-1) T, t) \times \Omega)}^{2}\left|\tilde{\chi}^{\left(k_{n}\right)}\right|_{3, \infty, \Omega^{t}}^{2} \\
+\left|d_{1}\right|_{L_{\infty}\left(((k-n-1) T, t) \times S_{2}\right)}^{2}\left|\tilde{\chi}^{\left(k_{n}\right)}\right|_{2, S_{2}^{t}}^{2} .
\end{aligned}
$$

We bound the fourth integral on the r.h.s. of (4.6) by

$$
\left|\chi^{\prime\left(k_{n}\right)}\right|_{10 / 3, \Omega^{t}}\left|F_{3}^{\left(k_{n}\right)}\right|_{10 / 7, \Omega^{t}}
$$

and the last term by

$$
\frac{1}{T^{2}}\|\chi-\tilde{\chi}\|_{L_{2}(((k-n-1) T, t) \times \Omega)}^{2} \leq \frac{1}{T^{2}} l_{1}^{2}((k-n-1) T, t) .
$$

Utilizing the above estimates in (4.6) we obtain

$$
\begin{aligned}
\left|\chi^{\prime\left(k_{n}\right)}(t)\right|_{2, \Omega}^{2} & +\int_{(k-n-1) T}^{t}\left\|\chi^{\prime\left(k_{n}\right)}\left(t^{\prime}\right)\right\|_{1, \Omega}^{2} d t^{\prime} \\
\leq & c\left(\varepsilon\left(\left|\chi^{\prime\left(k_{n}\right)}\right|_{10 / 3, \Omega^{t}}^{2}+\left|\nabla \chi^{\prime\left(k_{n}\right)}\right|_{2 ; \Omega^{t}}^{2}\right)\right. \\
& +\|\nabla v\|_{L_{2}(((k-n-1) T, t) \times \Omega)}^{2}\left|\tilde{\chi}^{\left(k_{n}\right)}\right|_{3, \infty, \Omega^{t}}^{2} \\
& +l_{1}^{2}((k-n-1) T, t)+\left\|d_{1}\right\|_{L_{\infty}\left(((k-n-1) T, t) \times S_{2}\right)}^{2}\left|\tilde{\chi}^{\left(k_{n}\right)}\right|_{2, S_{2}^{t}}^{2} \\
& \left.+\left|h^{\left(k_{n}\right)}\right|_{5, \Omega^{t}}^{2}\|\nabla v\|_{L_{2}(((k-n-1) T, t) \times \Omega)}^{2}+\left|F_{3}^{\left(k_{n}\right)}\right|_{10 / 7, \Omega^{t}}^{2}\right) .
\end{aligned}
$$


Since $\chi^{\prime}=\chi-\tilde{\chi}$, for sufficiently small $\varepsilon>0$ we have

$$
\begin{aligned}
& \left|\chi^{\left(k_{n}\right)}(t)\right|_{2, \Omega}^{2}+\int_{(k-n-1) T}^{t}\left\|\chi^{\left(k_{n}\right)}\left(t^{\prime}\right)\right\|_{1, \Omega}^{2} d t^{\prime} \\
& \leq c\left(\|\nabla v\|_{L_{2}(((k-n-1) T, t) \times \Omega)}^{2}\left|\tilde{\chi}^{\left(k_{n}\right)}\right|_{3, \infty, \Omega^{t}}^{2}\right. \\
& \quad+\left\|d_{1}\right\|_{L_{\infty}\left(((k-n-1) T, t) \times S_{2}\right)}^{2}\left|\tilde{\chi}^{\left(k_{n}\right)}\right|_{2, S_{2}^{t}}^{2} \\
& \quad+\left|h^{\left(k_{n}\right)}\right|_{5, \Omega^{t}}^{2}\|\nabla v\|_{L_{2}(((k-n-1) T, t) \times \Omega)}^{2}+\left|\tilde{\chi}^{\left(k_{n}\right)}\right|_{2, \infty, \Omega^{t}}^{2} \\
& \left.\quad+l_{1}^{2}((k-n-1) T, t)+\int_{(k-n-1) T}^{t}\left\|\tilde{\chi}^{\left(k_{n}\right)}\left(t^{\prime}\right)\right\|_{1, \Omega}^{2} d t^{\prime}+\left|F_{3}^{\left(k_{n}\right)}\right|_{10 / 7, \Omega^{t}}^{2}\right) .
\end{aligned}
$$

Now using the inequalities

$$
|u|_{10 / 3, \Omega^{t}} \leq c\left(|u|_{2, \infty, \Omega^{t}}+\|u\|_{L_{2}\left(0, t ; W_{2}^{1}(\Omega)\right)}\right) \leq c\|u\|_{s, r, \Omega^{t}},
$$

where $5 / r-3 / 2 \leq s$, and Lemma 2.1 we obtain

$$
\begin{aligned}
\mid \chi^{\left(k_{n}\right)}(t) & \left.\right|_{2, \Omega^{t}} ^{2}+\int_{(k-n-1) T}^{t}\left\|\chi^{\left(k_{n}\right)}\left(t^{\prime}\right)\right\|_{1, \Omega}^{2} d t^{\prime} \\
\leq & c\left(l_{1}^{2}((k-n-1) T, t)\left|\tilde{\chi}^{\left(k_{n}\right)}\right|_{3, \infty, \Omega^{t}}^{2}+l_{1}^{2}((k-n-1) T, t)\right. \\
& +l_{1}^{2}((k-n-1) T, t)\left|h^{\left(k_{n}\right)}\right|_{5, \Omega^{t}}^{2} \\
& \left.+\left(1+\left\|d_{1}\right\|_{L_{\infty}\left(((k-n-1) T, t) \times S_{2}\right)}^{2}\right)\left\|\tilde{\chi}^{\left(k_{n}\right)}\right\|_{s, r, \Omega^{t}}^{2}+\left|F_{3}^{\left(k_{n}\right)}\right|_{10 / 7, \Omega^{t}}^{2}\right) .
\end{aligned}
$$

Using the inequalities

$$
\left\|\tilde{\chi}^{\left(k_{n}\right)}\right\|_{s, r, \Omega^{t}} \leq c\left\|\chi_{*}^{\left(k_{n}\right)}\right\|_{s-1 / r, r, S_{1}^{t}} \leq\left\|v^{\left(k_{n}\right)}\right\|_{s, r, \Omega^{t}}
$$

and

$$
\left|\tilde{\chi}^{\left(k_{n}\right)}\right|_{3, \infty, \Omega^{t}} \leq\left|v^{\left(k_{n}\right)}\right|_{3, \infty, S_{1}^{t}},
$$

we obtain (4.5). This concludes the proof.

Next we consider the problem

$$
\begin{array}{ll}
v_{1, x_{2}}-v_{2, x_{1}}=\chi & \text { in } \Omega^{\prime}, \\
v_{1, x_{1}}+v_{2, x_{2}}=-h_{3} & \text { in } \Omega^{\prime}, \\
v^{\prime} \cdot n^{\prime}=0 & \text { on } S_{1}^{\prime},
\end{array}
$$

where $\Omega^{\prime}=\Omega \cap\left\{x_{3}=\right.$ const $\left.\in(-a, a)\right\}, S_{1}^{\prime}=S_{1} \cap\left\{x_{3}=\right.$ const $\left.\in(-a, a)\right\}$, and $x_{3}, t$ are treated as parameters. 
Lemma 4.2. Let the asumptions of Lemma 4.1 be satisfied. Then

$$
\begin{aligned}
& \left\|v^{\prime\left(k_{n}\right)}(t)\right\|_{1, \Omega}^{2}+\left\|\nabla v^{\prime\left(k_{n}\right)}\right\|_{L_{2}\left(0, t, W_{2}^{1}(\Omega)\right)}^{2} \\
& \leq c\left(l_{1}^{2}((k-n-1) T, t)\left(\left|h^{\left(k_{n}\right)}\right|_{5, \Omega^{t}}^{2}+1\right)\right. \\
& \quad+\left(1+\left|d_{1}\right|_{L_{\infty}\left(((k-n-1) T, t) \times S_{2}\right)}^{2}\right)\left\|v^{\prime\left(k_{n}\right)}\right\|_{s, r, \Omega^{t}}^{2} \\
& \left.\quad+\left|F_{3}^{\left(k_{n}\right)}\right|_{10 / 7, \Omega^{t}}^{2}+\left|h^{\prime\left(k_{n}\right)}\right|_{2, \infty, \Omega^{t}}^{2}+\int_{(k-n-1) T}^{t}\left\|h^{\prime\left(k_{n}\right)}\left(t^{\prime}\right)\right\|_{1, \Omega}^{2} d t^{\prime}\right) \\
& \equiv\left(A^{\left(k_{n}\right)}((k-n-1) T, t)\right)^{2}, \quad t \in((k-n-1) T,(k+1) T) .
\end{aligned}
$$

Proof. For solutions of problem (4.7) we get the estimates

$$
\begin{aligned}
\left\|v^{\prime\left(k_{n}\right)}\right\|_{1, \Omega^{\prime}}^{2} & \leq c\left(\left|\chi^{\left(k_{n}\right)}\right|_{2, \Omega^{\prime}}^{2}+\left|h_{3}^{\left(k_{n}\right)}\right|_{2, \Omega^{\prime}}^{2}\right), \\
\left\|v^{\prime\left(k_{n}\right)}\right\|_{2, \Omega^{\prime}}^{2} & \leq c\left(\left\|\chi^{\left(k_{n}\right)}\right\|_{1, \Omega^{\prime}}^{2}+\left\|h_{3}^{\left(k_{n}\right)}\right\|_{1, \Omega^{\prime}}^{2}\right),
\end{aligned}
$$

where $v^{\prime}=\left(v_{1}, v_{2}\right)$. Integrating the above estimates with respect to $x_{3}$ and the second one also with respect to time, and adding the results, we obtain

$$
\begin{aligned}
\int_{-a}^{a}\left\|v^{\prime\left(k_{n}\right)}\left(x_{3}, t\right)\right\|_{1, \Omega^{\prime}}^{2} d x_{3}+\int_{(k-n-1) T}^{t} \int_{-a}^{a}\left\|v^{\prime\left(k_{n}\right)}\left(x_{3}, t^{\prime}\right)\right\|_{2, \Omega^{\prime}}^{2} d x_{3} d t^{\prime} \\
\leq c\left(\int_{(k-n-1) T}^{t}\left\|\chi^{\left(k_{n}\right)}\left(t^{\prime}\right)\right\|_{1, \Omega}^{2} d t^{\prime}\right. \\
\left.\quad+\int_{(k-n-1) T}^{t}\left\|h_{3}^{\left(k_{n}\right)}\left(t^{\prime}\right)\right\|_{1, \Omega}^{2} d t^{\prime}+\left|\chi^{\left(k_{n}\right)}(t)\right|_{2, \Omega}+\left|h_{3}^{\left(k_{n}\right)}(t)\right|_{2, \Omega}^{2}\right) .
\end{aligned}
$$

In view of the above estimates we obtain

$$
\begin{aligned}
\left\|v^{\prime\left(k_{n}\right)}\right\|_{1, \Omega}^{2}+\int_{(k-n-1) T}^{t} & \left\|v^{\prime\left(k_{n}\right)}\left(t^{\prime}\right)\right\|_{2, \Omega}^{2} d t^{\prime} \\
\leq & c\left(\int_{(k-n-1) T}^{t}\left\|\chi^{\left(k_{n}\right)}\left(t^{\prime}\right)\right\|_{2, \Omega}^{2} d t^{\prime}+\left|\chi^{\left(k_{n}\right)}\right|_{2, \infty, \Omega^{t}}^{2}\right. \\
& \left.+\left|h^{\left(k_{n}\right)}\right|_{2, \infty, \Omega^{t}}^{2}+\int_{(k-n-1) T}^{t}\left\|h^{\left(k_{n}\right)}\left(t^{\prime}\right)\right\|_{1, \Omega}^{2} d t^{\prime}\right) .
\end{aligned}
$$

Applying (4.5) to estimate the first two norms in the last inequality and the inequality $\left|v^{\prime\left(k_{n}\right)}\right|_{3, \infty, S_{1}^{t}} \leq \varepsilon\left\|v^{\prime\left(k_{n}\right)}\right\|_{L_{\infty}\left(0, t, H^{1}(\Omega)\right)}+c(1 / \varepsilon)\left|v^{\left(k_{n}\right)}\right|_{2, \infty, \Omega^{t}}$ we obtain (4.8). This concludes the proof. 
Now we increase the regularity of $v$. Let us consider problem (1.1) in the form

$$
\begin{aligned}
& v_{, t}^{\left(k_{n}\right)}-\operatorname{div} \mathbb{T}\left(v^{\left(k_{n}\right)}, p^{\left(k_{n}\right)}\right)=-v^{\prime} \cdot \nabla v^{\left(k_{n}\right)}-w^{\left(k_{n}\right)} h \\
& +f^{\left(k_{n}\right)}+\xi_{, t}^{\left(k_{n}\right)} v \quad \text { in } \Omega^{T}, \\
& \operatorname{div} v^{\left(k_{n}\right)}=0 \\
& \text { in } \Omega^{T} \text {, } \\
& v^{\left(k_{n}\right)} \cdot \bar{n}=0 \\
& \text { on } S_{1}^{T} \text {, } \\
& \nu \bar{n} \cdot \mathbb{D}\left(v^{\left(k_{n}\right)}\right) \cdot \bar{\tau}_{\alpha}+\gamma v^{\left(k_{n}\right)} \cdot \bar{\tau}_{\alpha}=0, \quad \alpha=1,2, \\
& \text { on } S_{1}^{T} \text {, } \\
& v^{\left(k_{n}\right)} \cdot \bar{n}=d^{\left(k_{n}\right)} \\
& \text { on } S_{2}^{T} \text {, } \\
& \bar{n} \cdot \mathbb{D}\left(v^{\left(k_{n}\right)}\right) \cdot \bar{\tau}_{\alpha}=0, \quad \alpha=1,2, \\
& \text { on } S_{2}^{T} \text {, } \\
& \left.v^{\left(k_{n}\right)}\right|_{t=(k-n-1) T}=0 \\
& \text { in } \Omega \text {. }
\end{aligned}
$$

For this problem we obtain the inequality

$$
\begin{aligned}
& \left\|v^{\left(k_{n}\right)}\right\|_{2,5 / 3, \Omega^{t}}+\left|\nabla p^{\left(k_{n}\right)}\right|_{5 / 3, \Omega^{t}} \\
& \leq c\left(\left|v^{\prime} \cdot \nabla v^{\left(k_{n}\right)}\right|_{5 / 3, \Omega^{t}}+\left|w^{\left(k_{n}\right)} h\right|_{5 / 3, \Omega^{t}}+\frac{1}{T}\|v\|_{L_{5 / 3}(((k-n-1) T, t) \times \Omega)}\right. \\
& \quad+\left\|d_{1}^{\left(k_{n}\right)}\right\|_{7 / 5,5 / 3, S_{2}^{t}}+\left\|\gamma v^{\left(k_{n}\right)} \cdot \bar{\tau}_{\alpha}\right\|_{2 / 3,5 / 3, S_{1}^{t}}+\left|f^{\left(k_{n}\right)}\right|_{5 / 3, \Omega^{t}} .
\end{aligned}
$$

We estimate the first term of the r.h.s. by

$$
\left|v^{\prime} \cdot \nabla v^{\left(k_{n}\right)}\right|_{5 / 3, \Omega^{t}} \leq\left|v^{\left(k_{n+1}\right)}\right|_{10, \Omega^{t}}\left|\nabla v^{\left(k_{n}\right)}\right|_{2, \Omega^{t}},
$$

and the second term by

$$
\left|w^{\left(k_{n}\right)} h\right|_{5 / 3, \Omega^{t}} \leq\left|w^{\left(k_{n}\right)}\right|_{10 / 3, \Omega^{t}}\left|h^{\left(k_{n+1}\right)}\right|_{10 / 3, \Omega^{t}} .
$$

Using the above estimates and

$$
\left\|v^{\left(k_{n}\right)}\right\|_{2 / 5,5 / 3, S_{1}^{t}} \leq \varepsilon\left\|v^{\left(k_{n}\right)}\right\|_{2,5 / 3, \Omega^{t}}+c(1 / \varepsilon)\left|v^{\left(k_{n}\right)}\right|_{2, \Omega^{t}},
$$

we obtain the inequality

$$
\begin{aligned}
& \left\|v^{\left(k_{n}\right)}\right\|_{2,5 / 3, \Omega^{t}}+\left|\nabla p^{\left(k_{n}\right)}\right|_{5 / 3, \Omega^{t}} \\
& \leq \quad\left(A^{\left(k_{n+1}\right)}((k-n-2) T, t) l_{1}((k-n-1) T, t)+\left\|d_{1}^{\left(k_{n}\right)}\right\|_{7 / 5,5 / 3, S_{2}^{t}}\right) \\
& \left.\quad+\left(1+\left|h^{\left(k_{n+1}\right)}\right|_{10 / 3, \Omega^{t}}\right) l_{1}((k-n-1) T, t)+\left|f^{\left(k_{n}\right)}\right|_{5 / 3, \Omega^{t}}\right) .
\end{aligned}
$$

Using (4.8) and the inequality

$$
\left\|v^{\left(k_{n}\right)}\right\|_{s, r, \Omega^{t}} \leq \varepsilon\left\|v^{\left(k_{n}\right)}\right\|_{2,5 / 3, \Omega^{t}}+c(1 / \varepsilon)\left|v^{\left(k_{n}\right)}\right|_{2, \Omega^{t}},
$$


with $1+s<5 / r$, in (4.10), we obtain, for $t \in((k-n-1) T,(k+1) T)$,

$$
\begin{aligned}
\left\|v^{\left(k_{n}\right)}\right\|_{2,5 / 3, \Omega^{t}} \leq c\left(l _ { 1 } ( ( k - n - 1 ) T , t ) \left(l _ { 1 } ( ( k - n - 1 ) T , t ) \left(\left|h^{\left(k_{n+1}\right)}\right|_{5, \Omega^{t}}\right.\right.\right. \\
\left.\quad+\left|d_{1}\right|_{L_{\infty}\left(((k-n-1) T, t) \times S_{2}\right)}\right)+\left|F_{3}^{\left(k_{n+1}\right)}\right|_{10 / 7, \Omega^{t}} \\
\left.\quad+\left\|h^{\left(k_{n+1}\right)}\right\|_{V_{2}^{0}\left(\Omega^{t}\right)}\right)+\left|f^{\left(k_{n}\right)}\right|_{5 / 3, \Omega^{t}} \\
\left.\quad+\left(1+\left|h^{\left(k_{n+1}\right)}\right|_{10 / 3, \Omega^{t}}\right) l_{1}((k-n-1) T, t)+\left\|d_{1}^{\left(k_{n}\right)}\right\|_{7 / 5,5 / 3, S_{2}^{t}}\right) \\
\equiv A^{\prime\left(k_{n+1}\right)}((k-n-1) T, t) .
\end{aligned}
$$

Next for problem (4.9) we obtain the inequality

$$
\begin{aligned}
\left\|v^{\left(k_{n}\right)}\right\|_{2,2, \Omega^{t}}+\left|\nabla p^{\left(k_{n}\right)}\right|_{2, \Omega^{t}} \leq & c\left(\left|v^{\prime} \cdot \nabla v^{\left(k_{n}\right)}\right|_{2, \Omega^{t}}+\left|w^{\left(k_{n}\right)} h\right|_{2, \Omega^{t}}\right. \\
& +\frac{1}{T}|v|_{L_{2}(((k-n-1) T, t) \times \Omega)}+\left\|d_{1}^{\left(k_{n}\right)}\right\|_{3 / 2,2, S_{2}^{t}} \\
& \left.+\left\|\gamma v^{\left(k_{n}\right)} \cdot \bar{\tau}_{\alpha}\right\|_{1 / 2,2, S_{1}^{t}}+\left|f^{\left(k_{n}\right)}\right|_{2, \Omega^{t}}\right) .
\end{aligned}
$$

We estimate the first term on the r.h.s. by

$$
\left|v^{\prime} \cdot \nabla v^{\left(k_{n}\right)}\right|_{2, \Omega^{t}} \leq\left|v^{\left(k_{n+1}\right)}\right|_{10, \Omega^{t}}\left|\nabla v^{\left(k_{n}\right)}\right|_{5 / 2, \Omega^{t}},
$$

and the second term by

$$
\left|w^{\left(k_{n}\right)} h\right|_{2, \Omega^{t}} \leq\left|w^{\left(k_{n}\right)}\right|_{5, \Omega^{t}}\left|h^{\left(k_{n+1}\right)}\right|_{10 / 3, \Omega^{t}} .
$$

Using the inequalities

$$
\begin{aligned}
& \left|\nabla v^{\left(k_{n}\right)}\right|_{5 / 2, \Omega^{t}}+\left|v^{\left(k_{n}\right)}\right|_{5, \Omega^{t}} \leq c\left\|v^{\left(k_{n}\right)}\right\|_{2,5 / 3, \Omega^{t}}, \\
& \left\|v^{\left(k_{n}\right)}\right\|_{1 / 2,2, S_{1}^{t}} \leq \varepsilon\left\|v^{\left(k_{n}\right)}\right\|_{2,2, \Omega^{t}}+c(1 / \varepsilon)\left|v^{\left(k_{n}\right)}\right|_{2, \Omega^{t}},
\end{aligned}
$$

we obtain

$$
\begin{aligned}
\left\|v^{\left(k_{n}\right)}\right\|_{2,2, \Omega^{t}}+ & \left|\nabla p^{\left(k_{n}\right)}\right|_{2, \Omega^{t}} \leq c\left(\| v ^ { ( k _ { n } ) } \| _ { 2 , 5 / 3 , \Omega ^ { t } } \left(\left|v^{\prime\left(k_{n+1}\right)}\right|_{10, \Omega^{t}}\right.\right. \\
+ & \left.\left|h^{\left(k_{n+1}\right)}\right|_{10 / 3, \Omega^{t}}\right)+\left|f^{\left(k_{n}\right)}\right|_{2, \Omega^{t}}+l_{1}((k-n-1) T, t) \\
& \left.+\left\|d_{1}^{\left(k_{n}\right)}\right\|_{3 / 2,2, S_{2}^{t}}\right) .
\end{aligned}
$$

Now using in (4.12) the inequality

$$
\begin{aligned}
\left|v^{\prime\left(k_{n+1}\right)}\right|_{10, \Omega^{t}} \leq c\left(l _ { 1 } ( ( k - n - 1 ) T , t ) \left(\left|h^{\left(k_{n+1}\right)}\right|_{5, \Omega^{t}}\right.\right. \\
\left.+\left|d_{1}\right|_{L_{\infty}\left(((k-n-1) T, t) \times S_{2}\right)}+1\right)+\left|F_{3}^{\left(k_{n+1}\right)}\right|_{10 / 7, \Omega^{t}}+\left\|h^{\left(k_{n+1}\right)}\right\|_{V_{2}^{0}\left(\Omega^{t}\right)} \\
\left.+A^{\prime\left(k_{n+2}\right)}((k-n-3) T, t)\right) \equiv B^{\left(k_{n+2}\right)}((k-n-3) T, t),
\end{aligned}
$$

we obtain 
Lemma 4.3. Assume that $v \in W_{5 / 3}^{2,1}(\Omega \times((k-n-1) T, t))$. Then

$$
\begin{aligned}
\left\|v^{\left(k_{n}\right)}\right\|_{2,2, \Omega^{t}} & +\left|\nabla p^{\left(k_{n}\right)}\right|_{2, \Omega^{t}} \\
\leq & c\left(A ^ { \prime ( k _ { n + 1 } ) } ( ( k - n - 2 ) T , t ) \left(B^{\left(k_{n+2}\right)}((k-n-3) T, t)\right.\right. \\
& \left.+\left|h^{\left(k_{n+1}\right)}\right|_{10 / 3, \Omega^{t}}\right)+\left|f^{\left(k_{n}\right)}\right|_{2, \Omega^{t}}+l_{1}((k-n-1) T, t) \\
& \left.+\left\|d_{1}^{\left(k_{n}\right)}\right\|_{3 / 2,2, S_{2}^{t}}\right) .
\end{aligned}
$$

LEMmA 4.4. Let $v \in W_{2,2}^{2,1}(\Omega \times((k-n-2) T, t))$ and $g \in L_{2}(\Omega \times$ $((k-n-1) T, t))$. Then any solution of $(3.1)$ satisfies

$$
\begin{aligned}
\left\|h^{\left(k_{n}\right)}\right\|_{2,2, \Omega^{t}}+ & \left|\nabla q^{\left(k_{n}\right)}\right|_{2, \Omega^{t}} \leq c\left(\varphi\left(\left\|v^{\left(k_{n+1}\right)}\right\|_{2,2, \Omega^{t}}\right)\left|h^{\left(k_{n}\right)}\right|_{2, \Omega^{t}}\right. \\
& \left.+l_{1}((k-n-1) T, t)+\left\|d_{1, x}^{\left(k_{n}\right)}\right\|_{3 / 2,2, S_{2}^{t}}+\left|g^{\left(k_{n}\right)}\right|_{2, \Omega^{t}}\right),
\end{aligned}
$$

where $\varphi$ is an increasing positive function.

Proof. From (3.1) we get

$$
\begin{aligned}
\left\|h^{\left(k_{n}\right)}\right\|_{2,2, \Omega^{t}}+\left|\nabla q^{\left(k_{n}\right)}\right|_{2, \Omega^{t}} & \\
\leq & c\left(\left|v \cdot \nabla h^{\left(k_{n}\right)}\right|_{2, \Omega^{t}}+\left|h^{\left(k_{n}\right)} \cdot \nabla v\right|_{2, \Omega^{t}}+\frac{1}{T}|h|_{2, \Omega \times((k-n-1) T, t}\right) \\
& \left.\quad+\left\|\gamma h^{\left(k_{n}\right)} \cdot \bar{\tau}_{\alpha}\right\|_{1 / 2,2, S_{1}^{t}}+\left\|d_{1, x}^{\left(k_{n}\right)}\right\|_{3 / 2,2, S_{2}^{t}}+\left|g^{\left(k_{n}\right)}\right|_{2, \Omega^{t}}\right) .
\end{aligned}
$$

Using the Hölder inequality in (4.15) we obtain

$$
\begin{aligned}
& \left\|h^{\left(k_{n}\right)}\right\|_{2,2, \Omega^{t}}+\left|\nabla q^{\left(k_{n}\right)}\right|_{2, \Omega^{t}} \leq c\left(\left|v^{\left(k_{n+1}\right)}\right|_{10, \Omega^{t}}\left|\nabla h^{\left(k_{n}\right)}\right|_{5 / 2, \Omega^{t}}\right. \\
& \quad+\left|h^{\left(k_{n}\right)}\right|_{5, \Omega^{t}}\left|\nabla v^{\left(k_{n+1}\right)}\right|_{10 / 3, \Omega^{t}}+\frac{1}{T} l_{1}((k-n-1) T, t)+\left\|h^{\left(k_{n}\right)}\right\|_{1 / 2,2, S_{1}^{t}} \\
& \left.\quad+\left\|d_{1, x}^{\left(k_{n}\right)}\right\|_{3 / 2,2, S_{2}^{t}}+\left|g^{\left(k_{n}\right)}\right|_{2, \Omega^{t}}\right) .
\end{aligned}
$$

Now using the inequalitites

$$
\begin{aligned}
\left\|h^{\left(k_{n}\right)}\right\|_{1 / 2,2, S_{1}^{t}} \leq \varepsilon\left\|h^{\left(k_{n}\right)}\right\|_{2,2, \Omega^{t}}+c(1 / \varepsilon)\left|h^{\left(k_{n}\right)}\right|_{2, \Omega^{t}}, & \\
\left|v^{\left(k_{n+1}\right)}\right|_{10, \Omega^{t}}\left|\nabla h^{\left(k_{n}\right)}\right|_{5 / 2, \Omega^{t}} \leq & \varepsilon\left\|h^{\left(k_{n}\right)}\right\|_{2,2, \Omega^{t}}+\varphi_{1}\left(\left|v^{\left(k_{n+1}\right)}\right|_{10, \Omega^{t}, 1 / \varepsilon}\right)\left|h^{\left(k_{n}\right)}\right|_{2, \Omega^{t}}, \\
\left|\nabla v^{\left(k_{n+1}\right)}\right|_{10 / 3, \Omega^{t}}\left|h^{\left(k_{n}\right)}\right|_{5, \Omega^{t}} \leq & \varepsilon\left\|h^{\left(k_{n}\right)}\right\|_{2,2, \Omega^{t}} \\
& +\varphi_{2}\left(\left|\nabla v^{\left(k_{n+1}\right)}\right|_{10 / 3, \Omega^{t}, 1 / \varepsilon}\right)\left|h^{\left(k_{n}\right)}\right|_{2, \Omega^{t}},
\end{aligned}
$$

where $\varphi_{1}, \varphi_{2}$ are increasing positive functions, we obtain (4.14). This concludes the proof. 
Finally, from (4.14), (4.13) and (4.2) we obtain

$$
\begin{aligned}
\| h^{\left(k_{n}\right)} & \|_{2,2, \Omega^{t}} \\
\leq & \varphi\left(\left\|h^{\left(k_{n+2}\right)}\right\|_{2,2, \Omega^{t}}, G((k-n-3) T, t)\right) \eta_{k_{n}}((k-n-1) T, t) \\
& +G^{\prime}((k-n-1) T, t),
\end{aligned}
$$

where

$$
\begin{aligned}
& G((k-n-3) T, t)=l_{1}((k-n-1) T, t)+\left\|d_{1}^{\left(k_{n+1}\right)}\right\|_{8 / 5,5 / 2, S_{2}^{t}} \\
& \quad+\left|f^{\left(k_{n+1}\right)}\right|_{5 / 2, \Omega^{t}}+\left|F_{3}^{\left(k_{n+2}\right)}\right|_{10 / 7, \Omega^{t}}+\left|d_{1}^{\left(k_{n}\right)}\right|_{3,6, S_{2}^{t}}+\left|d_{1}^{\left(k_{n}\right)}\right|_{3, \infty, S_{2}^{t}} \\
& \quad+\left|d_{1}\right|_{L_{\infty}(((k-n-1) T, t) \times \Omega)}, \\
& G^{\prime}((k-n-1) T, t)=\left|g^{\left(k_{n}\right)}\right|_{2, \Omega^{t}}+l_{1}((k-n-1) T, t)+\left\|d_{1, x}^{\left(k_{n}\right)}\right\|_{3 / 2,2, S_{2}^{t} .} .
\end{aligned}
$$

Proof of Theorem 1.1. Let $T_{0}=3 T$. In view of [1], $\|h\|_{W_{2}^{2,1}\left(\Omega^{t}\right)} \leq A$, where $A>0$ is a sufficiently large constant. We choose $k=3$ and $n=0$ (when $n=0$ we write $k$ instead of $k_{0}$ ). Then (4.16) implies

$$
\left\|h^{(3)}\right\|_{W_{2}^{2,1}\left(\Omega^{t}\right)} \leq \varphi\left(A+\left\|h^{(3)}\right\|_{W_{2}^{2,1}\left(\Omega^{t}\right)}, G(0, t)\right) \eta_{3}(2 T, t)+G^{\prime}(2 T, t) .
$$

For $\eta_{3}(t)$ sufficiently small,

$$
\varphi\left(A+\left\|h^{(3)}\right\|_{W_{2}^{2,1}\left(\Omega^{t}\right)}, G(0, t)\right) \eta_{3}(2 T, t)+G^{\prime}(2 T, t) \leq A \quad \text { and } \quad G^{\prime}(t) \leq A .
$$

Hence $\left\|h^{(3)}\right\|_{W_{2}^{2,1}\left(\Omega^{t}\right)} \leq A$.

Assume now that for $n=0$ and $1 \leq m \leq s \in \mathbb{N}$ we have $\left\|h^{(m)}\right\|_{W_{2}^{2,1}\left(\Omega^{t}\right)}$ $\leq A$. We will show that

$$
\left\|h^{(s+1)}\right\|_{W_{2}^{2,1}\left(\Omega^{t}\right)} \leq A .
$$

From (4.16) it follows that

$$
\begin{aligned}
\left\|h^{(s+1)}\right\|_{W_{2}^{2,1}\left(\Omega^{t}\right)} \leq & \varphi\left(\left\|h^{(s-2)}\right\|_{W_{2}^{2,1}\left(\Omega^{t}\right)}+\left\|h^{(s-1)}\right\|_{W_{2}^{2,1}\left(\Omega^{t}\right)}+\left\|h^{(s)}\right\|_{W_{2}^{2,1}\left(\Omega^{t}\right)}\right. \\
& \left.+\left\|h^{(s+1)}\right\|_{W_{2}^{2,1}\left(\Omega^{t}\right)}, G((s-2) T, t)\right) \eta_{s+1}(s T, t)+G^{\prime}((s T, t) \\
\leq & \varphi\left(3 A+\left\|h^{(s+1)}\right\|_{W_{2}^{2,1}\left(\Omega^{t}\right)}, G((s-2) T, t)\right) \eta_{s+1}(s T, t) \\
& +G^{\prime}(s T, t) .
\end{aligned}
$$

If $\eta_{s+1}(s T, t)$ is small enough then (4.17) holds. This concludes the proof.

Acknowledgments. The author thanks Prof. W. Zajączkowski for very fruitful discussions during the preparation of this paper.

This research was partially supported by the Polish KBN Grant 1/P03A/ $021 / 30$. 


\section{References}

[1] P. Kacprzyk, Global regular nonstationary flow for the Navier-Stokes equations in a cylindrical pipe, Appl. Math. (Warsaw) 34 (2007), 289-307.

[2] T. Kobayashi and W. M. Zajączkowski, On global motion of a compressible barotropic viscous fluid with boundary slip conditions, ibid. 26 (1999), 159-194.

[3] O. A. Ladyzhenskaya and V. A. Solonnikov, On some problems of vector analysis and generalized boundary value problems for the Navier-Stokes equations, Zap. Nauchn. Sem. LOMI 59 (1976), 81-116 (in Russian).

[4] O. A. Ladyzhenskaya, V. A. Solonnikov and N. N. Ural'tseva, Linear and Quasilinear Equations of Parabolic Type, Nauka, Moscow, 1967 (in Russian).

[5] B. Nowakowski and W. M. Zajączkowski, Global existence of solutions to NavierStokes equations in cylindrical domains, Appl. Math. (Warsaw) 36 (2009), 169-182.

[6] J. Rencławowicz and W. M. Zajączkowski, Large time regular solutions to NavierStokes equations in cylindrical domains, Topol. Methods Nonlinear Anal. 32 (2008), 69-87.

[7] W. M. Zajączkowski, Long time existence of regular solutions to Navier-Stokes equations in cylindrical domains under boundary slip conditions, Studia Math. 169 (2005), 243-285.

[8] - Global existence of axially symmetric solutions to Navier-Stokes equations with large angular component of velocity, Colloq. Math. 100 (2004), 243-263.

[9] - Global special regular solutions to the Navier-Stokes equations in a cylindrical domain without the axis of symmetry, Topol. Methods Nonlinear Anal. 24 (2004), 60-105.

[10] - Global regular nonstationary flow for the Navier-Stokes equations in a cylindrical pipe, ibid. 26 (2005), 221-286.

[11] - Global special regular solutions to the Navier-Stokes equations in axially symmetric domains under boundary slip conditions, Dissertationes Math. 432 (2005).

[12] - Global special regular solutions to the Navier-Stokes equations in a cylindrical domain under boundary slip conditions, Gakuto Ser. Math. 21 (2004).

Institute of Mathematics and Cryptology

Cybernetics Faculty

Military University of Technology

Kaliskiego 2

00-908 Warszawa, Poland

E-mail: pkacprzyk@wat.edu.pl

Received on 30.1.2008;

revised version on 27.4.2009 